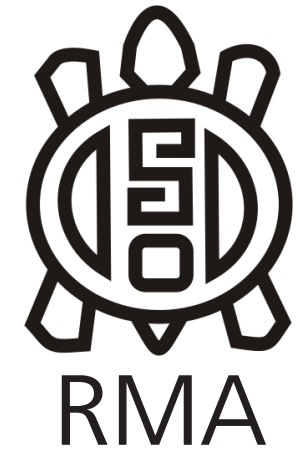

Dossier

\title{
Suelos, estratos, cosas
}

Soils, strata, things

Marco Alvarez*

*Agencia Nacional de Promoción Científica y Técnica (ANPCyT). Catedra de Geología del Cuaternario. División de mineralogía, petrología y sedimentología, División Arqueología, Facultad de Ciencias Naturales y Museo, Universidad Nacional de La Plata, Argentina. Paseo del bosque s/n (1900), La Plata.

E-Mail: Marco.alvarez@fcnym.unlp.edu.ar

\section{Resumen}

El siguiente ensayo busca ofrecer una alternativa para interpretar el registro sedimentario en arqueología, por consiguiente, concebiremos a los suelos, estratos y sucesiones sedimentarias como cosas. Las cosas son un lugar donde varios caminos se cruzan y se entrelazan, son un nudo compuesto por múltiples hilos que se pueden rastrear más allá del nudo en sí. Esta forma interpretativa, nos invita a reflexionar que todo aquello que envuelve a los materiales arqueológicos no es sólo una clase dentro de la taxonomía sedimentaria, sino que suelos y estratos son el resultado de una compleja asamblea de actores. Esta herramienta heurística aporta información contextual del pasado, así como también puede utilizarse para la identificación de elementos de interés con el fin de ampliar el horizonte de la investigación arqueológica. Luego de la definición y discusión de una serie de términos como suelos, estratos, objetos y cosas, interpretaremos la secuencia sedimentaria del sitio Cárcava Paso del Hervidero como cosa. Por último, se genera un ensamble compuesto por los múltiples elementos que componen a la cosa-secuencia del sitio arqueológico y dejaremos expuestas las hebras que guiarán investigaciones futuras.

Palabras clave: Geoarqueología; Cosas; Río uruguay; Teoría; Ensambles.

\begin{abstract}
The following essay seeks to offer an alternative to interpret the sedimentary record in archeology, where soils, strata and sedimentary successions are conceived as things. Things are a place where various paths intersect and intertwine, they are a knot made up of multiple threads that can be traced beyond the knot itself. In line with this, this interpretive form invites us to reflect that everything that surrounds archaeological materials is not only a class within the sedimentary taxonomy, but that soils and strata are the result of a complex assembly of actors. This heuristic tool provides contextual information from the past, as well as can also be used to identify elements of interest in order to broaden the horizon of archaeological research. After the definition and discussion of a series of terms such as soils, strata, objects and things, we will interpret Cárcava Paso del Hervideros site's sedimentary sequence as a thing. Finally, an assembly composed of the multiple elements that shape the thing-sequence of the archaeological site is generated and we will expose the strands that will guide future research.
\end{abstract}

Keywords: Geoarcheology; Things; Uruguay river; Theory; Assemblies.

\section{Introducción}

"El mundo habitado no se compone de objetos, sino de cosas"

(Ingold, 2010, p. 1)

Desde hace dos mil años, tal como enuncia Tim Ingold en su trabajo titulado Trayendo las cosas a la vida (Ingold, 2010), en el mundo occidental existe un modelo hilomórfico de pensar a las cosas, de cómo se hacen y cómo se utilizan. Este modelo entiende a las cosas como formas impuestas por un agente sobre una materia inerte, e ignora que la relación esencial, en un mundo repleto de vida, no es entre la forma y la materia, sino entre los materiales y las fuerzas (Deleuze y Guattari, 2004). Para derrocar este modelo, Ingold sugiere olvidarse de la materia y de las formas finales, y desarrollar una ontología en la cual se prioricen los procesos de formación, de flujo y de transformación de los materiales. Para ello, menciona una serie de definiciones en las que incluye que "...el mundo habitado no se compone de objetos, sino de cosas..." (Ingold, 2010, p. 1), debido a que los objetos se nos presentan como un hecho final, mientras que las cosas están sucediendo junto con nosotros (Heidegger, 1971).

Con el fin de resaltar que el mundo está compuesto por cosas, Ingold intenta concebir un escenario en el que no 
nos hallemos rodeados por estas. Para ello precisa alejarse de los muebles, la computadora, los lentes y todo aquello que ofrece su oficina. Al apartarse de su despacho, se imagina caminando por un bosque tupido y mientras observa a su alrededor, detecta rocas, animales, plantas, árboles y ramas. Un árbol, por ejemplo, ¿es un objeto o una cosa? se pregunta nuestro caminante, entonces fija la mirada en las partes del árbol (e.g., corteza, ramas, hojas, tronco), en los habitantes que viven en cada una de ellas (insectos, roedores, aves y hongos, etc.), en sus superficies, sus límites y las relaciones del árbol con su entorno (el aire y el viento). El autor concluye que "...él árbol no es un objeto en absoluto, sino una cierta reunión conjunta de las hebras de la vida..." (Ingold, 2010, p. 3) y eso es justamente, una cosa.

Desprendiéndose de la consideración de árbol-cosa, Ingold sostiene que todo lo que nos rodea son cosas, su despacho está lleno de cosas, las piedras son cosas, las nubes son cosas, y así cualquier entidad imaginable. Entonces, surge la pregunta ¿existe algún escenario en el cual no estemos rodeados por cosas? Ingold citando a Gibson (1979) nos da a entender que la única forma posible de estar ausente de las cosas es: estar desnudo en una superficie, en donde veamos el horizonte hacia cualquier lado donde miremos, donde el cielo se encuentre totalmente despejado y que la superficie, sobre la cual estamos parados, se encuentre desprovista de cualquier tipo de vegetación. Sólo en ese paisaje inhabitado e inhabitable (Ingold, 2010, p. 6) estaríamos por fin librados de las cosas.

Es en este punto en el cual deseo que nos detengamos, que miremos hacia nuestros pies (o los pies de Gibson), y resaltar que ni siquiera en ese escenario imaginario nos libraríamos de las cosas, dado que estaríamos obviando que nos encontramos parados por encima de una reunión conjunta de las hebras de la vida, una asamblea de materiales en movimiento, el resultado de varios caminos que se entrelazaron, un conjunto de sedimentos dispuestos de forma específica debido a múltiples agentes a lo largo del tiempo. Es decir que la superficie sobre la que estamos parados es una cosa, y es justamente, la superficie más fundamental de todas (Gibson, 1979; Ingold, 2010).

El objetivo por el cual escribo este ensayo es ofrecer otra forma de interpretar el registro sedimentario de los sitios arqueológicos. Proponemos pensar a los suelos y estratos como cosas, dado que ello nos abrirá las puertas a reflexionar que todo aquello que envuelve a los materiales arqueológicos no es sólo un nombre dentro de la taxonomía sedimentaria, ni un objeto o artefacto acabado y final, sino que es el resultado de una compleja asamblea de actores, compuesta por todos los componentes del sitio.

Esta herramienta heurística nos puede aportar información contextual del pasado, así como también es útil para el devenir de la investigación arqueológica. En el camino de denominar y entender a las cosas, puede ser utilizado en diferentes programas de investigación, como por ejemplo en la ecología histórica, la arqueología del paisaje y cualquier otra corriente que se encuentre interesada en las relaciones entre el ambiente y los grupos humanos en largos ciclos de tiempo.

\section{Aclarando los términos ¿Qué es un suelo, un estrato, un artefacto, un objeto y una cosa?}

Más allá que el lector pueda tener una idea de lo que significa cada una de estas palabras, es necesario aclarar de qué forma y cómo se entienden los términos en el contexto de este ensayo, sobre todo debido a que muchas veces suelen utilizarse como sinónimos. Además de las definiciones, se discutirán tres concepciones de los suelos y se disertará sobre las ventajas y desventajas de entender a cualquier entidad como artefactos, objetos y cosas.

\section{Suelos y estratos}

El término suelo es entendido y/o utilizado coloquialmente de tres formas. La primera de ellas es para nombrar una superficie firme por la cual podemos caminar, construir una casa, donde se desarrolla la vegetación, etc. La segunda es la forma que la edafología y pedología denominan a un cuerpo natural, formado a través de pedogénesis y compuesto por materia orgánica, minerales, líquidos y gases. Los suelos ocupan un espacio específico entre la superficie y las profundidades de la corteza terrestre y a la vez, se caracterizan por tener horizontes definidos, colores, texturas, estructuras y límites (Butzer, 1989; Harris; 1991; Comité Argentino de Estratigrafía, 1992; Soil Survey Staff, 2014). Los suelos se forman a través de la descomposición de una roca base o un sedimento parental, por agentes biológicos (e.g plantas, lombrices, hormigas) y meteorización química (e. g disolución, oxidación, hidratación e hidrólisis). Este proceso de formación de suelos se llama pedogénesis y se favorece de los momentos de estabilidad ambiental, lo que permite la ocupación humana (Castiñeira et al., 2019). En este trabajo, la primera de las concepciones de la palabra suelo será llamada superficie; y cuando hablemos de suelos estaremos haciendo referencia, justamente, a la segunda definición.

La tercera forma en la que comúnmente es utilizada la palabra suelo es para denominar indiscriminadamente a todos los sedimentos que se encuentran en la subsuperficie. Es necesario destacar que no todo sedimento subterráneo es suelo, sino que además de ellos existen los estratos o capas. Estos sinónimos son la unidad de menor categoría de las rocas sedimentarias; son de origen natural (marino, eólico, fluvial, glaciar), antrópicos o antropogénicos (Comité Argentino de Estratigrafía, 1992; Butzer, 1989; Harris, 1989; Castiñeira et al., 2014). 
Los estratos o capas, son clasificados según el tipo de estratificación que poseen, junto a sus características texturales y depositacionales. Una sucesión, o grupos, de estratos puede constituir un miembro o una formación, de forma tal que el término estrato (o estratos) puede ser reemplazado por Miembros o Formación (Comité Argentino de Estratigrafía, 1992; Benito-Calvo et al., 2015). También, los estratos pueden ser el basamento para la formación de suelos.

La concepción de que todos los sedimentos de la subsuperficie componían una masa homogénea prevaleció desde los inicios de la arqueología. No obstante, ha transcurrido un proceso de diálogo donde se han incorporado numerosos aportes, provenientes de las ciencias de la tierra hacia la arqueología que han dejado atrás estas nociones (Schiffer, 1972; Butzer, 1989; Harris, E., 1991; Harris, O., 1993; Dubois, 2009; Castiñeira, 2008; Castiñeira et al., 2014; D'Amore, 2007; Benito Calvo et al., 2015; Tchilinguirian et al., 2016). Fue gracias a estas contribuciones que se han desarrollado múltiples metodologías de análisis sedimentarios y estratigráficos que han enriquecido las investigaciones y resultados arqueológicos.

Ahora bien, ¿para qué sirven las definiciones previas? En un principio para dimensionar que los sedimentos que observamos en las excavaciones arqueológicas no son una masa homogénea y uniforme, sino que se encuentran compuestos por diferentes materiales, que poseen sus propios nombres y disposiciones, diversos orígenes y transformaciones. A la vez es importante destacar que, en la labor de la arqueología, los materiales no son recolectados flotando en el aire, sino que son desenterrados y recuperados en una sucesión sedimentaria, la cual no debe de ser obviada en las descripciones e interpretaciones arqueológicas.

Este ensayo propone que los suelos y estratos deben de ser incorporados en la investigación arqueológica, pero no como un anexo a los materiales arqueológicos sino junto con el registro. Para esto se busca una concepción teórica que nos permita reflexionar sobre las secuencias sedimentarias ${ }^{1}$, sus elementos, las relaciones que las componen, los orígenes, los procesos, los restos materiales y las actividades humanas. Entonces, ¿cuál es la concepción teórica que proponemos y la que mejor se ajusta para nuestro planteo?

\section{Suelo como artefacto}

Una de las concepciones teóricas que ha sido utilizada en el último tiempo es la de "suelos como artefactos". Esta idea surge a partir de Daigle (1996) (en Sampietro, 2007). El autor considera que los suelos son una vía para la comprensión de las sociedades, y que si se

1 Secuencia sedimentaria: la forma de denominar al conjunto materiales heterogéneos (suelos, estratos, capas, minerales, formaciones, etc.). consideran a estos como un artefacto se podría realizar técnicas comparativas con ellos. Esto es, categorizarlos tipológicamente, tecnológicamente y cronológicamente en relación a su tecno-función y socio-función (de la misma forma que se hacen con los artefactos) a través de análisis químicos entre suelos utilizados por humanos y no utilizados, junto con la correlación entre anomalías químicas asociadas (Sampietro, 2007).

Esta concepción, y su utilización, resulta fructífera para algunos investigadores, pero bajo el criterio que deseo optar, no es suficiente. En primera instancia, si partiéramos de esa premisa, nos estaríamos focalizando solamente en los suelos naturales y antrópicos, y como ya mencioné no sólo existen los suelos en las secuencias sedimentarias, sino que también existen los estratos. Por otra parte, la concepción clásica de artefacto nos invita a pensar en algo de origen antrópico, formado por un conjunto de piezas y fabricado con un fin determinado; lo cual quizás sería correcto para aquellos suelos creados, efectivamente, por seres humanos (e.g., suelos para cultivo, terrazas, cerritos, terras pretas) pero que no nos invita a dimensionar los procesos naturales, ni los múltiples agentes que intervienen en la formación y preservación de los mismos. Además de ello, no se estaría incluyendo a los materiales arqueológicos al estudio del suelo, los cuales varias veces son modificados por los procesos pedogenéticos y también pueden modificar el suelo (Zarate, 1994; Dubois, 2009).

\section{Suelos y estratos como objetos}

"...Yo toco con el pie una piedra en el camino. Seguramente, usted dirá, la piedra es un objeto..." (Ingold, 2010, p.3) plantea Ingold durante su camino por el bosque, y a partir de ello diserta que la piedra sería un objeto sí, y sólo sí, pudiéramos extraerle artificialmente de ella los procesos ígneos o sedimentarios que la originaron, los procesos de erosión que le dieron forma y los procesos de transporte que la llevaron a ese punto (Ingold, 2010). Dado que lo anterior es imposible, la piedra no es un objeto en lo absoluto, ya que comprender algo como objeto es tomarlo por lo que es, por una forma inanimada, completa y final, a la que el observador se enfrenta como un hecho consumado (Ingold, 2012). A la vez se estaría invisibilizando todos los procesos que hicieron que sea lo que es. Un objeto se nos presenta completo y realizado, encerrado en sus superficies exteriores, límites y definiciones (Heidegger, 1971).

¿Qué sucede si entendemos al conjunto de suelos y estratos cómo un objeto? Abordarlos de esta manera ha llevado a comprender a las sucesiones sedimentarias de forma estática y acabada; el producto de esta concepción, en los trabajos arqueológicos, usualmente ha dado como resultado descripciones geológicas desconectadas del registro arqueológico, clasificaciones texturales, edades absolutas en las que un suelo se formó o agentes 
depositacionales de cierto estrato. En estos casos, no sólo, se está cajoneando (black-boxed) la interpretación (Shanks y Hodder, 1995), sino que al concebir a la secuencia como objeto no nos invita a pensar más allá del perfil en el sitio, más allá de la clasificación textural, del fechado o del agente sedimentario, así como tampoco nos permite rastrear las hebras que componen esa cosa.

Lo dicho hasta este punto no significa, ni intenta sostener, que el análisis de los objetos o artefactos empiece y termine sobre ellos mismos, o que no se hayan realizados aportes significativos sobre los tópicos que se discuten. Existen planteos como Laguens (2007), donde se propone pensar a los objetos de forma relacional, realizar una ruptura epistemológica y así construir una red casi infinita de relaciones de "objetos en objetos", y planteos relevantes e innovadores como E. Harris (1991) dónde crea un sistema de relaciones entre los artefactos, estratos sedimentarios y la estratigrafía en los sitios arqueológicos. Este texto se encuentra en línea con los anteriores antecedentes, pero aquí se propone emplear un concepto disruptivo, que al mencionarlo nos remita automáticamente a lo que queremos resaltar. Es decir, el término objeto o artefacto nos resulta insuficiente, entonces lo que se propone es que los suelos y estratos son cosas, formados por otras cosas.

\section{Las cosas}

Cosa es la traducción al español de la palabra thing proveniente del antiguo nórdico/inglés ping y del thing del antiguo alto alemán, la raíz etimológica de ambas palabras significa: asamblea, ensamblaje, reunión, duración (Olsen, 2003; Latour, 2004; Ingold, 2007, 2010). También, existe una posible raíz tenku, la cual sugiere que cosa significa: duración o tiempo extendido y estirado (Olsen, 2010, p.109). Entonces, cuando se utilice la palabra cosa se estará haciendo referencia a la traducción de ping y thing, y lo que debemos hacer es permitirnos reflexionar que aquello llamado cosa, es una conjunción, un ensamble, una asamblea, una reunión de partes que tiene un origen y una duración.

No existe una teoría dominante sobre la cosa (Ingold, 2012), sino más bien un espacio de debate, en el que muchas nociones diferentes de lo que puede ser cosa se pelean por obtener la atención (Gosden, 2005; Latour, 2004; Henare et al., 2007; Knappett, 2005, 2008; Bennett, 2010; Olsen 2010). Existen autores que utilizan cosa y objeto en modo de sinónimos (Olsen, 2010), otros que optan por no definir qué es una cosa, bajo el argumento de que las cosas deben de definirse por sí mismas (Henare et al., 2007, p. 4-5), y existe otro grupo que marca una profunda diferencia entre lo que es objeto y cosa (Heidegger; 1971; Ingold, 2010; Whitmore, 2014).

En línea con la anterior distinción, un objeto es una forma completa y final que se destaca por encima y en contra del perceptor, bloqueando un movimiento adicional (heurístico) y la correspondencia con el mismo (Ingold, 2012). En contraposición, la cosa es una reunión de materiales en movimiento, un particular anudar junto la materia-flujo (Heidegger, 1971). Concebir a algo como cosa es unirse a sus procesos de formación permanente, no sólo en el pasado, no sólo en el presente ni sólo en el futuro, sino en los tres tiempos a la vez. La cosa nos invita a tocarla, observarla y entender qué está sucediendo, que es un lugar donde varios caminos se cruzan y entrelazan. Ver una cosa no es sólo estar en frente de algo, sino es ser invitado a la reunión (Ingold, 2012).

También se puede pensar a las cosas como un "parlamento de líneas" (Ingold, 2007, p.5; Latour, 2004) donde la cosa es un nudo compuesto por hilos que se pueden rastrear más allá del nudo en sí, y en esa búsqueda, encontraremos que esos hilos forman parte de otros nudos.

Por último, con el fin de pensar cómo se definen, cómo son y el funcionamiento de las cosas, podemos incluir los lineamientos de Whitmore (2014), quien plantea que las cosas son ensambles, es decir, que configuran una reunión de elementos heterogéneos ensamblados por otras entidades. Los ensambles son una forma de pensar el mundo, que cambia nuestro modo de ver las apariencias finales de todos los elementos que existen, que se unen y que sostienen todas las cosas diferentes del mundo (Lucas, 2012). Estos ensambles, y por lo tanto las cosas, no son un todo sin fisuras, ni un conjunto que posea propiedades más relevantes que la suma de las partes que lo componen (De Landa, 2006).

\section{¿Qué cuenta cómo cosa?}

"El arado, la rueda, el molino, la mesa en que apoyo el vaso de vino, las curvas de la montaña rusa, la semicorchea y hasta la semifusa, el té, los ordenadores y los espejos, los lentes para ver de cerca y de lejos, la cucha del perro, la mantequilla, la yerba, el mate y la bombilla, el hambre y el sobrepeso, las curas de sueño, drogas de diseño y los perros adictos a las drogas en las aduanas. Hay tantas cosas, yo solo preciso dos, mi guitarra y vos" (Drexler, 2004).

Esta composición literaria de J. Drexler nos sirve cómo primera aproximación a identificar algunas de todas las cosas que existen, que incluye desde el arado hasta la persona a la que le dedica la canción. Todo es cosa y no existe nada que no pueda ser interpretado como cosa, ni las cosas hechas por humanos, ni las cosas pensadas por los humanos, ni los artefactos, ni los ecofactos, ni las cosas cinéticas, ni las inertes (Ingold, 2010, 2012; Whitmore, 2014). Puede suceder que el lector de este ensayo se encuentre dubitativo y escéptico con a la aceptación de que todo lo que nos rodean son cosas y 
que además, las cosas son una reunión de materiales, partes y/o factores en movimiento. Para acercarlo un poco más a la aceptación del término se debe de entender que lo que se propone es una concepción de la cosa-comoheurística y no como una cosa-como-analítica (Henare et al., 2007). Es decir, en vez de aplicar un conjunto de criterios teóricos predeterminados con los cuales medir las cosas (cosa analítica), le permitiremos a las cosas que se presenten a sí mismas y que sirvan como un método o una herramienta, con la cual podamos identificar un campo de fenómenos, los cuales después generaran teoría (cosa-heurística). A través de esta forma, podremos tallar las cosas, e identificar el campo desde el cual pueden emerger los criterios de evaluación de la cosa. "...Lo analítico analiza, lo heurístico meramente localiza..." (Henare et al., 2007, p.4).

Y si el lector aún sigue con desconfianza de que todo lo que nos rodea son cosas, me gustaría aclarar que este término no es una extrapolación de un término extranjero, si no que las cosas están presentes en nuestro dialecto desde nuestra niñez. Le invito a recordar el "veo veo $^{2 \prime}$, aquel juego de adivinanzas infantil y pasatiempos rutero, en donde la cosa que uno veía podía ir desde el celeste cielo, hasta el verde pasto, pasando por la marrón mancha del insecto sobre el parabrisas o la negra silueta del automóvil en la ruta. En el juego todo es cosa y esas cosas no son estáticas, es decir, tienen sus propiedades cambiantes. Por ejemplo, en el "veo veo", se puede decir que el cielo es celeste, gris si es un día lluvioso, rojo durante un atardecer y negro si es de noche, hasta incluso todas estas propiedades (o estados del cielo) pueden suceder durante el mismo viaje. Entender algo como cosa es detenerse, observarlo y no pensarlo en un estado estático e inerte, sino pensarlo como un ensamble de materiales en movimiento, con propiedades cambiantes, las cuales pueden ser rastreadas.

\section{Suelos y estratos como cosas}

Hemos mencionado que esta herramienta heurística nos invita pensar a cualquier cosa como una "reunión conjunta de hebras de la vida" (Ingold, 2010), "un ensamble de materiales en movimiento" (Heidegger, 1971), "una reunión de elementos heterogéneos ensamblados por otras entidades" (Whitmore, 2014). Pero ¿de qué forma se traduce esto a un estrato o un suelo? Para contestar esta pregunta se retoman las definiciones genéricas de estos cuerpos geológicos.

Al comienzo de este ensayo, se señaló que los suelos son el producto de la descomposición de un material base a partir de la acción de agentes biológicos y meteorización química. Dicho de otra forma, un agente natural depositó una serie de elementos heterogéneos,

2 "Veo, veo" es un juego de adivinanzas infantil que suele jugarse para pasar el tiempo en los viajes de ruta, en el cual se debe de descubrir qué cosa está observando uno de los participantes. Se juega a partir de un diálogo entre dos o más personas. los cuales sirvieron de base para la formación de un suelo a partir de la alteración, por parte de la vida, de diversos insectos y plantas, conjuntamente con las condiciones de temperatura y humedad. Es decir, los suelos son el resultado de múltiples eventos que han ocurrido en distintos lapsos temporales, con distintas magnitudes y escalas. Justamente esto es una reunión de elementos heterogéneos ensamblados por otras entidades, una reunión conjunta de las hebras de la vida, en fin, una cosa.

Por otra parte, mencionamos que un estrato es una unidad sedimentaria con características particulares y específicas, resultado de la erosión - transporte por parte de diversos agentes y la depositación de elementos heterogéneos que se encontraban en movimiento. Ahora, lo invito a pensar en un estrato, cualquiera sea, para luego identificar los orígenes, duraciones y su devenir, en otras palabras, reflexione sobre cuáles son los agentes, los procesos, los materiales implícitos y los resultantes. Por ejemplo, si pensamos en un estrato de conchillas marinas inserto en un acantilado, tendríamos que registrar, el mar, las tormentas, las mareas, las conchillas, la erosión, el carbonato de calcio, el pH, los insectos, el viento, las arenas, los acantilados, la corriente litoral, la humedad, etc. Lo que acabamos de lograr fue dejar al descubierto los hilos del nudo, hemos identificado algunos de todos los materiales y actores del estrato-cosa.

A una escala mayor, podemos pensar una sucesión sedimentaria compuesta por varios estratos y suelos, la cual llamaremos cosa - secuencia sedimentaria. Esta nueva cosa no es más que un ensamble más grande de numerosos materiales que ahora delimitan el campo heurístico en donde se basará la investigación arqueológica. Es así como, en el camino de pensar, delimitar y crear una cosa, iremos trazando las líneas de nuestra investigación.

\section{Aplicación de cosa}

Habiendo llegado al bagaje teórico mínimo de qué son las cosas (Henare, 2007, p.4) y al porqué entendemos a las secuencias sedimentarias como estas, utilizaremos esta herramienta heurística en el sitio arqueológico Cárcava Paso del Hervidero, ubicado en la cuenca media del río Uruguay. En esta sección presentaremos los datos que se han obtenido a partir de los primeros estudios arqueológicos y geoarqueológicos realizados; y a partir de ello se identificaran las distintas hebras que componen el ensamble.

\section{Sitio arqueológico "Cárcava Paso del Hervidero"}

El sitio arqueológico Cárcava Paso del Hervidero, se encuentra ubicado en la intersección del arroyo Hervidero y el río Uruguay, en la localidad de Puerto Yeruá, Entre Ríos, Argentina (figura 1) 
Figura 1: Ubicación del sitio Cárcava Paso del Hervidero.

Figure 1: Cárcava Paso del Hervidero's site ubication.

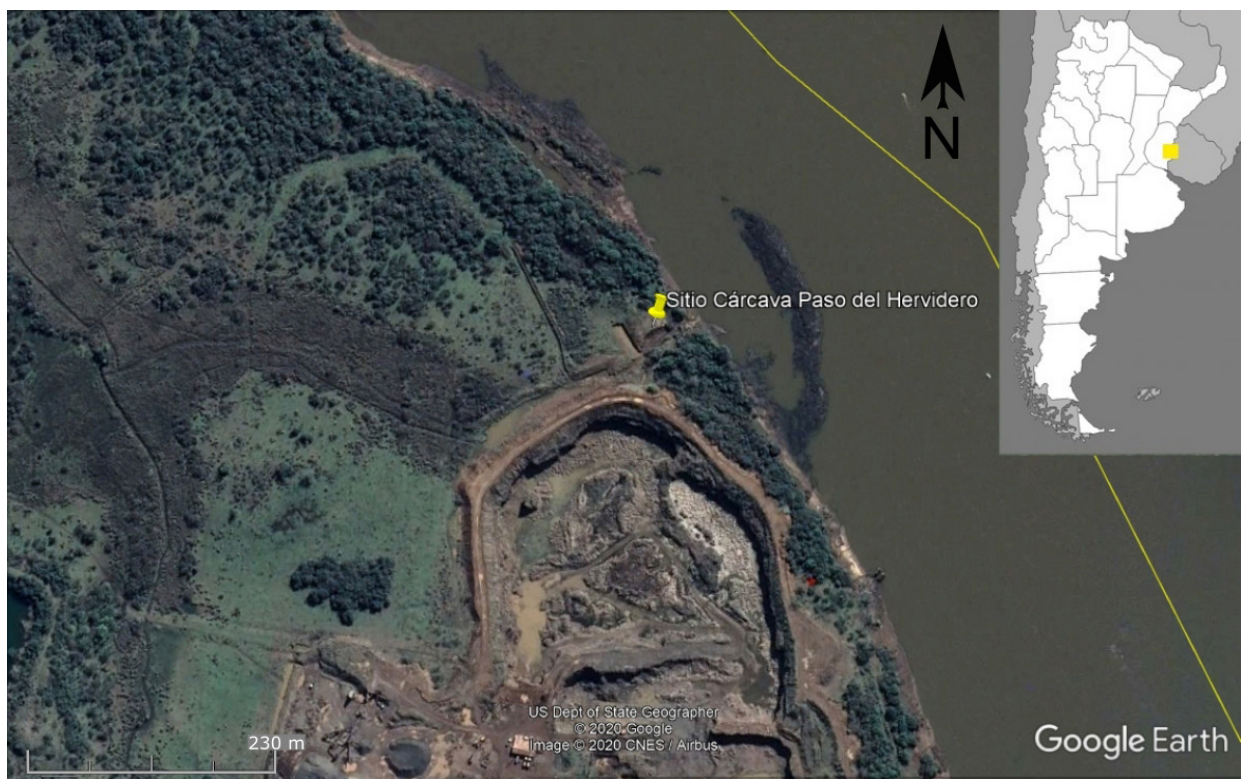

El sitio figura desde el 2013 como una localidad con potencial arqueológico (Rodríguez y Weissel, 2013). Desde ese entonces no se registran investigaciones en el área, hasta los últimos años donde el sitio ha sido retomado en los trabajos de campo realizados en la cuenca del río Uruguay en el marco de investigaciones interdisciplinarias y binacionales (Capdepont et al., 2017; Costa Angrizani, Zucol, Alvarez y Castiñeira, 2018a; Costa Angrizani et al., 2018b). En este contexto fue que en 2016, durante una campaña de prospección arqueológica, se hallaron numerosos materiales (líticos y cerámicos) en la superficie de la playa del río Uruguay y en la desembocadura de un arroyo (figura 2) (Castiñeira et al., 2019a). Luego del reconocimiento geomorfológico del área, se detectó que el material hallado en superficie provenía de una de las barrancas del arroyo, la cual fue profundizada a causa de la profundización artificial del arroyo para el desagote de una cantera que se encuentra en las proximidades (Capdepont et al., 2019).

El material en superficie fue recolectado, y luego se procedió a la observación, junto con la descripción de la secuencia sedimentaria de la barranca de dónde provenía el registro arqueológico. Sobre la barranca (figura 3) se detectaron 11 estratos sedimentarios diferentes a partir de su color, textura, estructuras y límites; además de ello, se determinó que el material arqueológico provenía de un paleosuelo ubicado a un metro de profundidad del techo de la barranca (Castiñeira et al., 2019a). Luego de ello, se tomaron muestras sedimentarias, en la totalidad de la secuencia estratigráfica, independientemente de que se haya determinado que el material estuviera proviniendo de sólo una de las 11 capas detectadas.

Los resultados de los estudios geoarquelógicos y los análisis texturales realizados sobre las muestras ${ }^{3}$ llevaron

3 La descripción estratigráfica se menciona de forma general debido a que no es la finalidad de este trabajo desarrollar las a agrupar las 11 capas en 3 unidades sedimentarias (figura 4), las cuales fueron descriptas desde la base al techo cómo: Unidad 1 con $2 \mathrm{~m}$ de espesor, compuesta por sedimentos fango arenosos masivos intercalados por lentes arenosos y areno fangosos, en el techo de esta primera unidad se detectó un primer paleosuelo de 30 $\mathrm{cm}$ de espesor. En contacto neto con la anterior unidad, apoya la Unidad 2, la cual presenta una potencia de 3 $\mathrm{m}$, formadas por niveles masivos fango arenosos, niveles areno fangosos, lentes arenosos de color castaño y un nivel pedogenético que culmina la secuencia, sobre este paleosuelo se realizó una datación radiocarbónica que dio como resultado un fechado de $950 \pm 50$ AP (LP 3441, 14C, materia orgánica) (Castiñeira et al., 2019 a y b). En este segundo suelo fósil se recuperó material arqueológico in-situ. Culminando la secuencia estratigráfica, se encuentra la Unidad 3, que posee 1,80 m de espesor y está representada por acumulaciones areno limosas a fangosas, por encima de esta se ubica la cobertura vegetal (Castiñeira et al., 2019a).

El análisis de la composición de los tiestos encontrados (total $n=31$ borde $n=5$ y cuerpo $n=26$ ) dio como resultado que, para la fabricación de cerámica, se utilizaron espículas de esponja de río denominada Uruguaya coralloides como antiplásticos; al observar en lupa macroscópica las muestras sedimentarias de la primer unidad sedimentaria, se detectaron las mismas espículas insertas en la matriz.

En cuanto a los materiales líticos muestreados y analizados ( $n=162$ ), se plantea que la tecnología utilizada estuvo orientada a la explotación de cantos rodados de rocas silíceas como calcedonia y areniscas y también de basaltos. Estas materias primas representan el $46,3 \%$, el $44,4 \%$ y el $7,4 \%$ de la muestra, respectivamente.

técnicas geoarqueológicas para la descripción y análisis de perfiles sedimentarios. 


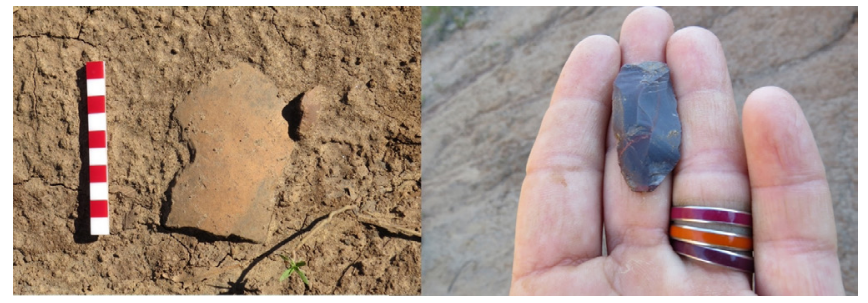

Figura 2: Material cerámico (Izquierda) y material lítico (derecha) recolectados en superficie.

Figure 2: Ceramic material (Left) and lithic material (Right) collected on the surface.

El análisis tecnológico permitió registrar técnica de talla de percusión unipolar directa para la reducción de núcleos y la producción de lascas. También se han detectado evidencias de diversas etapas de la secuencia de explotación de los rodados (eliminación de la corteza externa, extracción de lascas y manufactura de instrumentos) sobre lascas de filos bifaciales (Castiñeira et al., 2019a).

\section{Discusión de las cosas}

En base a los datos obtenidos en campo y laboratorio se procederá a enhebrar la información que compone la cosasecuencia sedimentaria. En un principio, los sedimentos fango arenosos de estructura masiva que conforman las dos primeras unidades y su tendencia granodecreciente, son interpretados geomorfológicamente como dos planicies de inundación superpuestas verticalmente. Estas representan numerosos eventos de desbordes del río Uruguay. Sobre estas dos unidades fluviales, se halla el paleosuelo datado en 950 + 50 años AP (LP 3441, 14C, Materia orgánica) (Castiñeira et al., 2019 a y b), que evidencia un periodo en donde cesaron las inundaciones del río. A la vez, este proceso de estabilidad ambiental se correlaciona con las condiciones climáticas imperantes durante el Período Cálido Medieval ca. 1400-800 años AP (Iriondo y Kröling, 2009).

De forma paralela al proceso pedogenético se produjo la ocupación humana de esa parte del litoral. Los ocupantes habitaron este nuevo espacio disponible, tuvieron aprovisionamiento local de los rodados para la confección de herramientas líticas y utilizaron las arenas junto con las arcillas disponibles para la fabricación de cerámica. Los desechos de estas actividades quedaron contenidas en el suelo que hoy compone el tope de la Unidad 2 de la secuencia.

El análisis de la tercera unidad sedimentaria, aquella que se encuentra suprayacente a la unidad que contiene el material arqueológico, dio como resultado que el estrato se encuentra conformado por sedimentos fango-arenosos depositados por el río Uruguay. Esto fue interpretado como una nueva crecida del río que impidió a los grupos permanecer en el sitio.

Por último, en el techo de la secuencia sedimentaria se encuentra el suelo actual. Se han realizado diferentes mediciones composicionales y de materia orgánica sobre el suelo actual y los valores obtenidos son similares al paleosuelo de la unidad 2, por lo que es posible imaginar que la superficie y las condiciones climáticas del presente sean las mismas o similares a las de 1000 años AP cuando sucedió la ocupación humana.

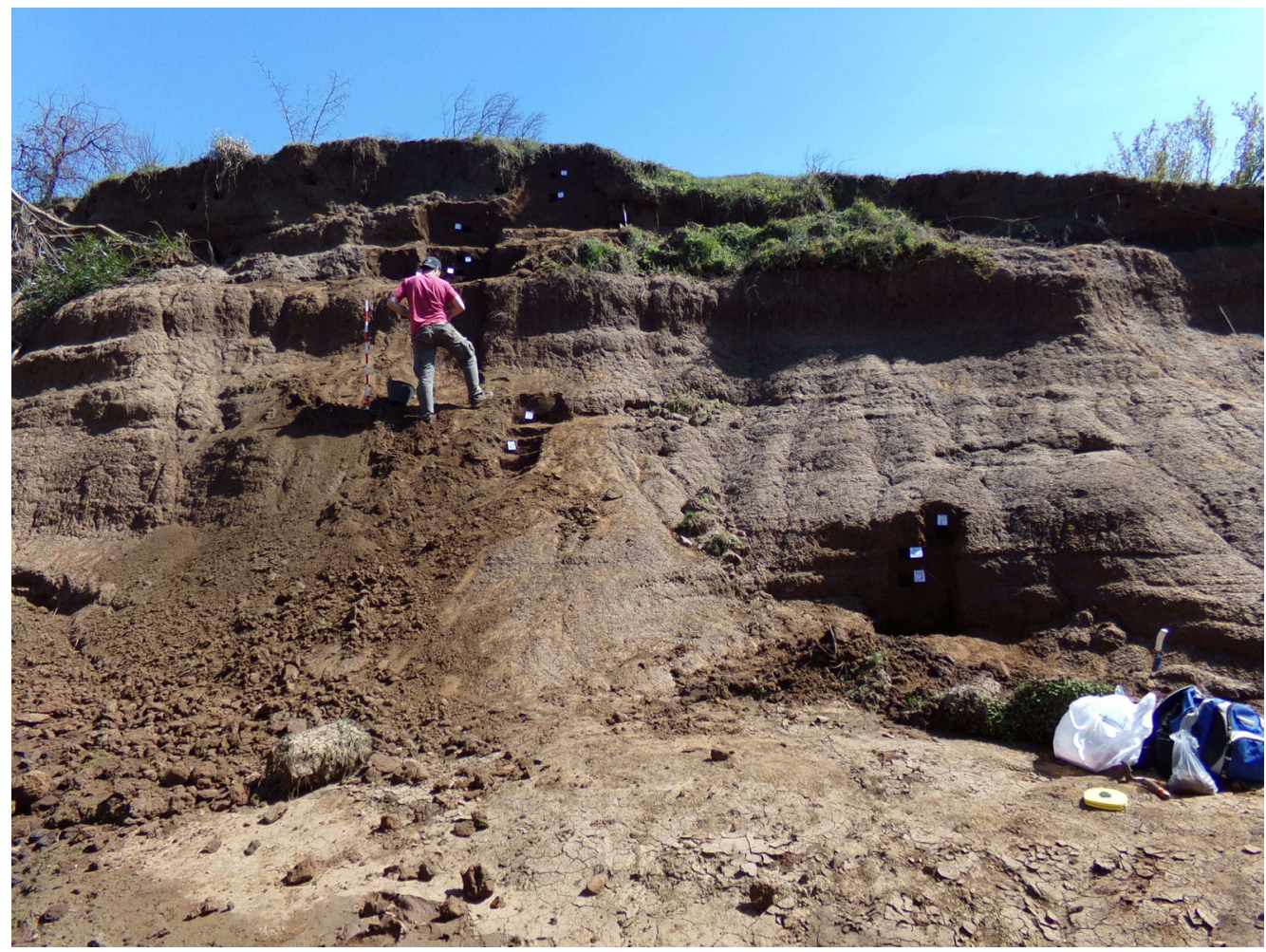

Figura 3: Secuencia sedimentaria del sitio Cárcava Paso del hervidero.

Figure 3: Cárcava Paso del Hervidero's sedimentary sequence. 


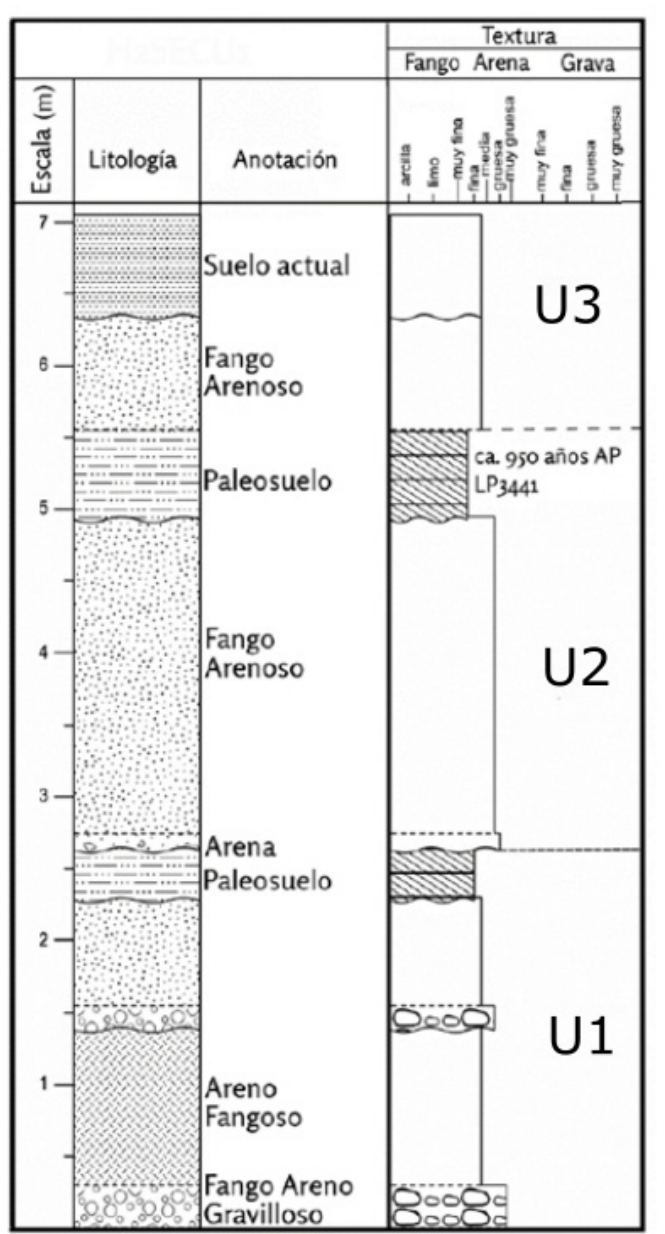

Figura 4: Esquema representativos de la secuencia de Cárcava Paso del Hervidero. (Tomado de Castiñeira et al. 2019)

Figure 4: Representative diagram of Cárcava Paso del Hervidero's sedimentary sequence. (From Castiñeira et al. 2019)

Todos los elementos que se han mencionado hasta este punto, son algunas de todas las hebras que forman y formaron la cosa-secuencia sedimentaria, es decir: el tiempo, el fechado, las espículas, el agua, las crecidas del río, el cálido medieval, los estratos, la vegetación, la materia orgánica, el arroyo, la profundización artificial, la barranca, los colores, estructuras y texturas, las gravas, fangos y arenas, la forma en que se mezclan, los restos líticos y los cerámicos, la biota, las decisiones humanas de ocupar en ese lugar, las ventajas que ofrece, el desarrollo de las actividades humanas, los aportes y alteraciones sedimentarias, el proceso pedogenético, etc. Cada uno de los registrados elementos la cosa-secuencia, los cuales se encuentran contenidos en los suelos y estratos de esa barranca de 7 metros de espesor.

A través de considerar a la secuencia sedimentaria como cosa nos hemos invitado a dejar de pensarla como un artefacto o un objeto acabado, estático y final. De ahora en más intentaremos concebirla en tanto conjunto de elementos que, de alguna forma, se encuentran entretejidos, relacionados y ensamblados. Cada uno de estos hilos de la cosa, ya identificados, son ahora nuevas líneas de evidencias cuyas hebras pueden ser perseguidas de acuerdo a nuestros temas de investigación, también podríamos escribir una historia con cada una de ellas, o realizarnos un sinfín de preguntas que nos abran las puertas a nuevas dimensiones heurísticas.

\section{Consideraciones finales}

Hacia el comienzo del trabajo, se deslizaron algunas palabras acerca de la posibilidad de la utilización e inclusión de la concepción de cosa en arqueología del paisaje y ecología histórica, o cualquier corriente teórica interesada en analizar, describir o hipotetizar la relación que existió entre los humanos del pasado y los ambientes que ocuparon. En virtud de ello considero que es posible la aplicación del término cosa sobre cualquiera que sea nuestro tema de interés, dado que invitará a reflexionar acerca del origen, el ser y el estar, conjuntamente a las relaciones que se establecen en ese nudo de la vida.

En cuanto a lo tratado propiamente en este ensayo, hemos aportado a la discusión sobre la importancia de los suelos y estratos en la investigación arqueológica. Además de ello, logramos concebir a estos cuerpos sedimentarios como cosas, lo que nos permitió identificar el gran conjunto materiales heterogéneos que los componen. La aplicación de esta herramienta heurística nos llevó a delimitar nuestro campo de fenómenos y le dimos cuerpo a todo un mundo de elementos que suceden en la cosa - secuencia.

Es así como se logró identificar algunos de todos los actores presentes en esa cosa - secuencia, los cuales ahora están presentes para ampliar nuestras investigaciones sobre el sitio. En línea con ello, una de las nuevas líneas que surgen a partir de este ensayo es que es imperioso profundizar en huellas tafonómicas de los materiales arqueológicos y sedimentarios para poder vincular la ocupación humana al desarrollo del paleosuelo, la selección del sector de ocupación y las condiciones climáticas regionales. Se deprende de lo anterior, reflexionar acerca de la existencia de metodologías específicas, o más idóneas, para la recolección de datos en el campo que nos lleven a identificar mayor cantidad de cosas y las relaciones entre ellas.

A través de pensar en las cosas, generamos un ensamble compuesto de suelos, estratos, crecidas del río, agentes naturales, características climáticas regionales, materiales arqueológicos, decisiones y acciones de los grupos humanos, que ha permitido identificar numerosos componentes para continuar el análisis del sitio Cárcava Paso del Hervidero.

La Plata, 5 de Agosto, 2020 


\section{Agradecimientos}

A Andres Laguens por la predisposición y la oportunidad de participar de este dossier. Al Dr. R. Angrizani, Dra. Carola Castiñeira y Dr. Eduardo Apolinaire por la lectura crítica y recomendaciones. A V. Romano, E. Mac Donagh y J. Fuertes por las sugerencias. Por último, a los revisores por sus valiosos aportes. Estas investigaciones cuentan con el apoyo financiero de la Agencia Nacional de Promoción Científica y Tecnológica (PICT 2017-2723) y (PICT 2014-0851).

\section{Bibliografía}

Benito-Calvo, A., Campaña Lozano, I., Karampaglidis, T. (2015). Conceptos básicos y métodos en geoarqueología: geomorfología, estratigrafía y sedimentología. Treballs d'Arqueologia, 20, 41. https://doi.org/10.5565/rev/tda.45

Bennett, J. (2010) Vibrant Matter: a political ecology of things, London: Duke University Press

Binford L. (1962) Archaeology as anthropology. American Antiquity 28: 217-225.

Butzer, K. W. (1989). Arqueología-Una ecología del hombre: Método y teoría para un enfoque contextual. Bellaterra.

Capdepont, I. C., Castiñeira, L. C., Costa Angrizani, R., Alvarez, M. (2019). Paleosuelos. Guías Para la Resolución Cronológica de la Variabilidad Climática y de las Ocupaciones Humanas en el Litoral del Río Uruguay. Geologia, 32(1), 105-125.

Capdepont, I., Castiñeira, L. C., Blasi, A. y del Puerto, L, (2017). Conectividad social durante el Holoceno tardío en el paisaje arqueológico del litoral oriental del bajo río Uruguay. Pesquisas Antropología, 73, 93-120.

Castiñeira, L. C., Blasi, A., Bonomo, M., Politis, G., \& Apolinaire, E. (2014). Modificacion antropica del paisaje durante el Holoceno tardío: Las construcciones monticulares en el delta superior del río Paraná, Argentina. Revista de La Asociacion Geologica Argentina, 71(1), 33-47.

Castiñeira, L. C., Costa Angrizani, R., Apolinaire, E., Alvarez, M., Capdepoint, C. I., Blasi, A., Maravilla, L., Mari, F., Zech, M., (2019a). Recod of late Holocene human ocupation in coastal deposit of the middle Uruguay river. Advances in coastal geoacheology in Latinamerica. Springer. (ISSN 2366-3421.)

Castiñeira, L. C., Capdepont, C. I., Costa Angrizani, R., Apolinaire, E., Alvarez, M., Blasi, A., Zucol, A. F. (2019b). Investigaciones arqueológicas en el tramo medio del Río Uruguay. Aportes para la caracterización de los escenarios paleoambientales durante el Holoceno tardío. Revista de Arqueología. Dossier septiembre-diciembre: 15-38.

Comité Argentino de Estratigrafía. (1992). Código Argentino de Estratigrafía. Serie B (Didactica y complementaria). Asociación Geológica Argentina.

Costa Angrizani, R., Zucol, A. F., Alvarez, M. y Castiñeira, L. C. (2018a). Reconstrucción de la dinámica paleoambienal de la cuenca media del río Uruguay durante el Holoceno tardío: análisis paleobotánicos y sedimentológicos. Boletín de la Asociación Latinoamericana de Paleobotánica y Palinología, 18, 99-100.

Costa Angrizani, R., Coll Moritan, V., Scabuzzo, C., Colobig, M. M., Zucol, A. F., Castiñeira L. C., Alvarez, M., Ramos, R. S. y Ramos van Raap, M. A. (2018b) Arqueologia no rio Uruguai: abordagens multidisciplinares para o estudo das dinámicas sociais e ambientais. Trabajo presentado en el XI Encontro da Sociedade de Arqueologia Brasileira-Sul, Curitiba, Brasil.

D'Amore, L. (2007). Narrar las prácticas del pasado. El potencial narrativo de la estratigrafía arqueológica como representativa de prácticas sociales. Intersecciones En Antropologia, 8, 101-119. ISSN 1666-2105

Deleuze, G. and F. Guattari (2004). A thousand plateaus, trans. B. Massumi. London: Continuum

DeLanda, M. (2006) A New Philosophy of Society: Assemblage Theory and Social Complexity, London: Continuum.

Diagle, R.P. (1996). Soil as artifact: Soil chemistry from the Levi Jordan plantation in Brazoria Country, Texas. Tesis de Maestría. Universidad de Huston. USA.

Drexler, J. (2004). Guitarra y vos. ECO. CD. Warner Music Latina.

Dubois, C. M. F. (2009). Geoarquelogía: Explorando propiedades espaciales y temporales del registro arqueológico. Perspectivas Actuales En Arqueología Argentina.

Gibson, J. J. (1979). The ecological approach to visual perception. Boston: Houghton Mifflin.

Gosden, C. (2005). What do objects want? Journal of Archaeological Method and Theory 12(3): 193-211.

Harris, E. C. (1991). Principios de estratigrafía arqueológica, 2nd edn. In Editorial Crítica Barcelona.

HARRIS, O.liver, J T. HARRIS. (1993). More More than representation: multi-scalar assemblages and the Deleuzian challenge to archaeology. History of the Human 
Sciences, Vol 31 (3): 2018. Traducción: Andrés Laguens, Octubre 2019

Henare, A., Holbraad, M., Wastell, S. (2007). Introducción: Pensar a través de las cosas. En Thinking through things. Theorising artefacts ethnographically. Editado por Amiria Henare, Martin Holbraad y Sari Wastell. Routledge, Londres, 2007. Cap. 1, pp. 1-31. [Traducción: Andrés Laguens, Diciembre de 2009].

Heidegger, M. (1971). Poetry, language, thought, trans. A. Hofstadter. New York: Harper \& Row.

Ingold, T. (2007). Materials against materiality. Archaeological Dialogues 14(1): 1-16.

Ingold, T. (2010). [Bringing Things to Life: Creative Entanglements in a World of Materials, en: Realities Working Papers. www.manchester.ac.uk/realities. Traducción: Andrés Laguens, Octubre 2011]

Ingold, T. (2012). Toward an Ecology of Materials. En: Annual Review of Anthropology 2012, vol. 41:427-42. Traducción: Andrés Laguens, febrero 2014].

Iriondo, M. H. y Kröhling, D. M. (2009). Cambios Ambientales en la cuenca del Río Uruguay. Desde dos millones de años hasta el Presente. Universidad Nacional del Litoral.

Knapett, C. (2005). Thinking through material culture. Philadelphia: University of Pennsylvania Press.

Laguens, A. (2007). Objetos en objetos. Hacia un análisis relacional de lo estético en Arqueología. Iconica Anqituitas. Bogotá: Universidad de Tolima. 2008 vol.2008 $\mathrm{n}^{\circ} \cdot \mathrm{p} 1-11$

Latour, B. (2004). Why has critique run out of steam? From matters of fact to matters of concern. Crit. Inq. 30:225-48.

Lucas, G. (2012). Entendiendo el registro arqueológico. Las entidades arqueológicas (Understanding the Archaeological Record, Cambridge University Press, Cap. 5: 169-214, 2012. Traductor: Andrés Laguens, Octubre de 2017).

Malafouris, L. and C. Knappett (2008). Material Agency. Springer
Olsen, B. (2003). Material Culture after Text: ReMembering Things" Norwegian Archaeological Review, Vol. 36, No. 2, 2003 [Traducción: Andrés Laguens, Agosto 2011].

Olsen, B. (2010). In Defense of Things. Plymouth, UK: Altamira.

Rodríguez, B. y Weissel, M. (2013). Exploraciones Arqueológicas. En A. Ten Hoeve, V. Fernández, M. López Bustigorri, C. Moreno, A. Morello, B. Rodríguez Basulto, M. Weissel, P. Cansanello, R. Orioli, S. Agostino, L. Navarro, M. Beber, C. Toselli, M. S. Pérsico, N. Galli y S. De la Madrid (Eds.), Recuperación del patrimonio hispánico guaraní en el corredor del río Uruguay (pp. 63-80). Buenos Aires: Consejo Federal de Inversiones de la Provincia de Entre Ríos. http://biblioteca.cfi.org.ar/ wpcontent/uploads/sites/2/2013/12/50747.pdf

Sampietro Vattuone, M., \& Marta. (2007). El suelo como artefacto: una experiencia desde el noa. Arqueometrá latinoamericana.

Shanks, M., Hodder I. (1995). Processual, postprocessual and interpretative archaeologies. En: Interpreting Archaeology. Finding meaning in the past, ed. porl. Hodder, M. Shanks, A Alexandri, V. Buchli, J. Carman, J. Last y G. Lucas. Routledge, 1995. Chap. 1: 3-29. Traducción: Andres Laguens.

Schiffer, Michael. (1972) Contexto Arqueológico y contexto sistémico. American Antiquity, vol 37, N²: pp.156-165.

Soil Survey Staff. (2014). Claves para la Taxonomía de Suelos. Mdp.Edu.Ar, 339. https://www.nrcs.usda. gov/Internet/FSE_DOCUMENTS/nrcs142p2_051546. pdf

Tchilinguirian, P., Ozan, I., Morales, M. (2016). Suelos y Geología Argentina. El suelo y la arqueología, Capítulo 9, p $252-396$

Witmore, C. (2014). La arqueología y los Nuevos Materialismos. [Archaeology and the New Materialisms. Journal of Contemporary Archaeology 1.2 (2014) 1-44. Traducción de Andrés Laguens, Septiembre 2017].

Zarate, M. (1994). Geoarqueología. Jornadas de arqueología e interdicisplinas (pp. 21-33). 